\title{
Characteristics and treatment patterns in older patients with locally advanced head and neck cancer (KCSG HN13-01)
}

Eun Joo Kang ${ }^{1,}{ }^{,}$, Yun-Gyoo Lee ${ }^{2, *}$, Bhumsuk Keam ${ }^{3}$, Jin-Hyuk Choi ${ }^{4}$, Jin-Soo Kim ${ }^{5}$, Keon Uk Park ${ }^{6}$, Kyoung Eun Lee ${ }^{7}$, Hyo Jung Kim ${ }^{8}$, Keun-Wook Lee ${ }^{9}$, Min Kyoung Kim ${ }^{10}$, Hee Kyung Ahn ${ }^{11}$, Seong Hoon Shin ${ }^{12}$, Jii Bum Lee ${ }^{13}$, Jung Hye Kwon ${ }^{14}$, Hye Ryun Kim ${ }^{13}$, Sung-Bae Kim ${ }^{15}$, and Hwan Jung Yun ${ }^{14}$

${ }^{1}$ Department of Internal Medicine, Korea University Guro Hospital, Seoul; ${ }^{2}$ Department of Internal Medicine, Kangbuk Samsung Hospital, Sungkyunkwan University School of Medicine, Seoul; ${ }^{3}$ Department of Internal Medicine, Seoul National University Hospital, Seoul; ${ }^{4}$ Department of Hematology-Oncology, Ajou University Hospital, Suwon; ${ }^{5}$ Department of Internal Medicine, Seoul Metropolitan Government Seoul National University Boramae Medical Center, Seoul; ${ }^{6}$ Department of Hemato-Oncology, Keimyung University Dongsan Medical Center, Daegu; ${ }^{7}$ Department of Hematology and Oncology, Ewha Womans University Mokdong Hospital, Seoul; ${ }^{8}$ Department of Internal Medicine, Hallym University Sacred Heart Hospital, Anyang; ${ }^{9}$ Department of Internal Medicine, Seoul National University Bundang Hospital, Seongnam; ${ }^{10}$ Department of Hematology-Oncology, Yeungnam University Medical Center, Daegu; ${ }^{11}$ Department of Internal Medicine, Gachon University Gil Medical Center, Incheon; ${ }^{12}$ Department of Internal Medicine, Kosin University Gospel Hospital, Busan; ${ }^{13}$ Department of Internal Medicine, Yonsei Cancer Center, Yonsei University College of Medicine, Seoul; ${ }^{14}$ Department of Internal Medicine, Chungnam National University Hospital, Daejeon; ${ }^{15}$ Department of Internal Medicine, Asan Medical Center, University of Ulsan College of Medicine, Seoul, Korea

\section{Characteristics and treatment patterns in older patients with locally advanced head and neck cancer}

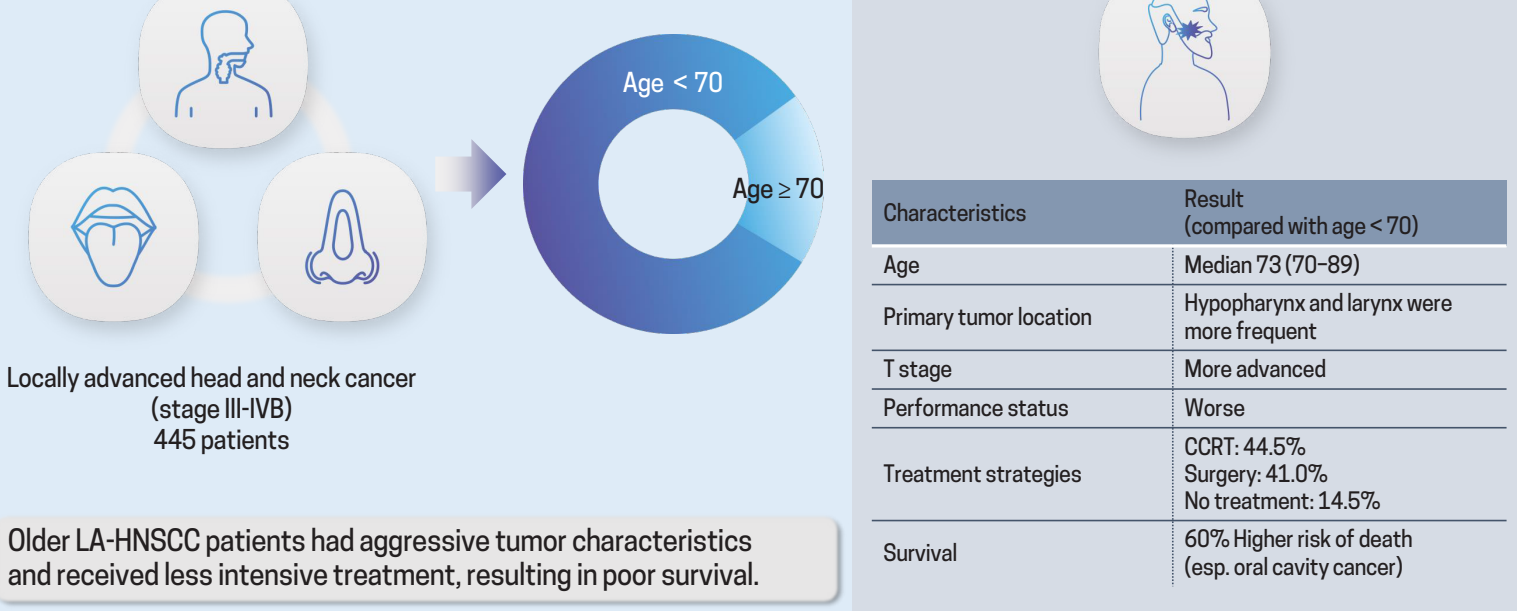

Received : November 30, 2020

Revised : June 10, 2021

Accepted: October 5, 2021

Correspondence to Hwan Jung Yun, M.D.

Department of Internal Medicine, Chungnam National University Hospital, 282 Munhwa-ro, Junggu, Daejeon 35015, Korea

Tel: +82-42-280-7157, Fax: +82-42-257-5753, E-mail: hjyun@cnuh.co.kr, https://orcid.org/00000003-0696-1235

\section{*These authors contributed equally to this work.}


Background/Aims: Treatment decisions for locally advanced head and neck squamous cell carcinoma (LA-HNSCC) are complicated, and multi-modal treatments are usually indicated. However, it is challenging for older patients to complete treatments. Thus, we investigated disease characteristics, real-world treatment, and outcomes in older LA-HNSCC patients.

Methods: Older patients (aged $\geq 70$ years) were selected from a large nationwide cohort that included 445 patients with stage III-IVB LA-HNSCC from January 2005 to December 2015. Their data were retrospectively analyzed and compared with those of younger patients.

Results: Older patients accounted for $18.7 \%$ (83/445) of all patients with median age was 73 years (range, 70 to 89 ). Proportions of primary tumors in the hypopharynx and larynx were higher in older patients and older patients had a more advanced T stage and worse performance status. Regarding treatment strategies of older patients, $44.5 \%$ of patients received concurrent chemoradiotherapy (CCRT), $41.0 \%$ underwent surgery, and $14.5 \%$ did not complete the planned treatment. Induction chemotherapy (IC) was administered to $27.7 \%$ (23/83) of older patients; the preferred regimen for IC was fluorouracil and cisplatin (47.9\%). For CCRT, weekly cisplatin was prescribed 3.3 times more often than 3-weekly cisplatin (62.2\% vs. $18.9 \%$ ). Older patients had a $60 \%$ higher risk of death than younger patients (hazard ratio, $1.6 ; p=0.035$ ). Oral cavity cancer patients had the worst survival probability.

Conclusions: Older LA-HNSCC patients had aggressive tumor characteristics and received less intensive treatment, resulting in poor survival. Further research focusing on the older population is necessary.

Keywords: Aged; Head and neck neoplasms; Therapeutics

\section{INTRODUCTION}

Head and neck squamous cell carcinoma (HNSCC) is a heterogeneous group of epithelial malignancies that arise from the sinus, oral cavity, oropharynx, hypopharynx, and larynx. HNSCC is the sixth most common malignancy worldwide, with 532,000 new cases every year [1]. In Korea, HNSCC is the tenth most common malignancy, with around 4,700 new cases each year and around one third of new cases occur in older patients with aged $\geq 70$ years $[2,3]$. HNSCC is common in the older population, and thus, its incidence is expected to increase soon in countries with an aged population, such as Korea $[4,5]$. Therefore, interests focusing on the treatment and care for older HNSCC patients. In particular, multi-modal treatment approaches have significant potential to result in better outcomes in locally advanced (LA)-HNSCC patients. Considering tumor and patient characteristics, LA-HNSCC patients are recommended to undergo surgery, radiotherapy, and chemotherapy, either sequentially or simultaneously.

However, compared with younger patients, older patients are more likely to be frail, and thus, aggressive treatment modalities can be dangerous [6]. Older patients also tend to have impaired functional status and more comorbidities, which can also affect treatment compliance. Furthermore, regarding the occurrence of treatment-related adverse events, older patients have a higher risk of treatment interruption or early discontinuation than younger patients, thus affecting survival and quality of life among the older population $[7,8]$. Therefore, proper treatment planning for older patients is warranted. Despite this, however, there are limited reports regarding tumor characteristics and treatment patterns in older LA-HNSCC patients. Thus, in this study, we focused on older patients (aged $\geq 70$ years) who were included in a large nationwide cohort of patients with stage III-IVB LA-HNSCC. This study aimed to investigate and compare differences in clinical characteristics, real-world treatment patterns, outcomes, and prognostic factors between older and younger patients.

\section{METHODS}

\section{Study population}

This large nationwide cohort included 445 patients with histologically proven LA-HNSCC from January 2005 to December 2015 from 13 nationwide referral hospitals in Korea (KCSG HN 13-01). LA-HNSCC was defined as clinical stage III-IVB based on the 7th edition of the American Joint Committee on Cancer Staging. Study patients aged $\geq 20$ 
years with primary squamous cell carcinoma of the oropharynx, hypopharynx, larynx, oral cavity, or nasal cavity were included irrespective of their treatment. Patients with pathologically confirmed squamous cell carcinoma of the cervical lymph node without primary site were regarded as having cancer of the head and neck origin and were also included in the analysis. We excluded patients with nasopharyngeal cancer, distant metastasis at initial diagnosis, or a history of other malignancies detected within 3 years of HNSCC diagnosis.

The chronological definition of the "older" has not yet been established. However, 70 years of age is considered a transitional point of senescent change, and therefore, 70 years of age is frequently used as a reference point in many clinical trials or geriatric evaluations in oncology $[9,10]$. Therefore, in this study, we defined "older" as those aged $\geq$ 70 years. Each participating hospital treated LA-HNSCC patients based on the principle of using multidisciplinary care treatment protocols. The study protocol was approved by the Institutional Review Board in main hospitals (Seoul National University Hospital and Chungnam National University Hospital: IRB-H-1304-089-481, 2013-10-003) and each participating center. All analyses were conducted retrospectively. Ethics Committee of each participated hospital approved this study without the need for individual subject consent.

\section{Study outcomes and statistical analysis}

The primary outcome of this study was to demonstrate the therapeutic patterns of real-world LA-HNSCC treatment among older patients and compare them with those of patients $<70$ years. The secondary outcome was to determine overall survival (OS) based on treatment strategy or primary site in older patients. OS was defined as the time from the date of HNSCC diagnosis to the date of death irrespective of the cause.

To compare patients' characteristics between groups, chi-square and independent $t$ test were used. Treatment response was assessed using RECIST version 1.1. Multivariate Cox regression analysis was used to determine risk factors for OS under the proportional hazards assumption. Two-sided $p$ value $<0.05$ indicated statistical significance. All statistical analyses were conducted using Stata version 16.0 software (StataCorp., College Station, TX, USA).

\section{RESULTS}

\section{Patient characteristics}

Among 445 LA-HNSCC patients who were examined during the study period, 83 (18.7\%) were older (age $\geq 70$ years) and had a median age of 73 years (range, 70 to 89). Approximately $93 \%$ ( $n=77$ ) of older patients were male, whereas among younger patients, only $85 \%(n=308)$ were male $(p=0.062)$. Older patients had worse performance status (PS) than younger patients $(p=0.014)$. History of smoking and alcohol intake were not different between older and younger patients. Primary tumor locations in older patients included the oropharynx in $26.5 \%$ of cases, followed by oral cavity $(24.1 \%)$, hypopharynx (17.0\%), larynx (18.1\%), and other sites (14.5\%). Other sites included the maxillary sinus, nasal cavity, ethmoid sinus, and head and neck squamous carcinoma with unknown primary tumor site. The proportion of older patients with tumor location in the hypophar$y n x$, larynx, and other sites was higher than that of younger patients, while the proportion of older patients with tumor location in the oropharynx was lower than that of younger patients $(p<0.001)$. Regarding T classification, higher proportion in older patients had T4a/b stage than younger patients $(p=0.018)$. Among 25 older patients who were tested for human papillomavirus (HPV) status, 40\% (10/25) were positive. Patients' demographic data are summarized in Table 1.

\section{Treatment strategy}

Treatment history for LA-HNSCC patients was divided into a definitive concurrent chemoradiotherapy (CCRT) group, surgery group, and inadequate group according to the decision of multidisciplinary team of each institution (Fig. 1). Among older patients $44.5 \%$ received definitive CCRT, whereas $41.0 \%$ underwent surgery. The remaining $14.5 \%$ did not receive any treatment or further adequate treatments after induction chemotherapy (IC) with the aim of cure such as CCRT or surgery. Meanwhile, $53.0 \%$ of patients aged $<70$ years received definitive CCRT, $42.3 \%$ underwent surgery, and $4.7 \%$ received inadequate treatment. The proportion of patients with inadequate treatment was approximately three times higher among older patients than among younger patients.

Regarding IC, $27.7 \%$ (23/83) of older patients received IC, whereas $37.2 \%(135 / 362)$ of younger patients received IC $(p=0.100)$. Among 83 older patients, eight $(9.6 \%)$ re- 
Kang EJ, et al. Treatment in older head and neck cancer

Table 1. Demographics in patients with locally advanced head and neck squamous cell carcinoma by age of 70

\begin{tabular}{|c|c|c|c|c|}
\hline \multirow{2}{*}{ Characteristic } & \multicolumn{2}{|c|}{ Age group, yr } & \multirow{2}{*}{$p$ value } & \multirow{2}{*}{$\begin{array}{c}\text { Total } \\
(n=445,100 \%)\end{array}$} \\
\hline & Age $\geq 70 \quad(n=83,18.7 \%)$ & Age $<70(n=362,81.3 \%)$ & & \\
\hline Age, yr & $73(70-89)$ & $57(24-69)$ & $<0.001$ & $61(24-89)$ \\
\hline Gender & & & 0.062 & \\
\hline Female & $6(7.2)$ & $54(14.9)$ & & $60(13.5)$ \\
\hline Male & $77(92.8)$ & $308(85.1)$ & & $385(86.5)$ \\
\hline ECOG PS & & & 0.014 & \\
\hline 0 & $3(3.6)$ & $52(14.4)$ & & $55(12.4)$ \\
\hline 1 & $40(48.2)$ & $175(48.3)$ & & $215(48.3)$ \\
\hline 2 & $6(7.2)$ & $11(3.0)$ & & $17(3.8)$ \\
\hline 3 & $2(2.4)$ & $2(0.6)$ & & $4(0.9)$ \\
\hline Unknown & $32(38.6)$ & $122(33.7)$ & & $154(34.6)$ \\
\hline Smoking history & & & 0.750 & \\
\hline Never & $16(19.3)$ & $83(22.9)$ & & $99(22.3)$ \\
\hline Former & $28(33.7)$ & $106(29.3)$ & & $134(30.1)$ \\
\hline Current & $18(21.7)$ & $89(24.6)$ & & $107(24.0)$ \\
\hline Unknown & $21(25.3)$ & $84(23.2)$ & & $105(23.6)$ \\
\hline Alcohol history & & & 0.747 & \\
\hline Do not drink & $25(30.1)$ & $95(26.2)$ & & $120(27.0)$ \\
\hline Drink alcohol & $30(36.1)$ & $133(36.8)$ & & $163(36.6)$ \\
\hline Unknown & $28(33.8)$ & $134(37.0)$ & & $162(36.4)$ \\
\hline Primary tumor location & & & $<0.001$ & \\
\hline Oropharynx & $22(26.5)$ & $169(46.7)$ & & $191(42.9)$ \\
\hline Oral cavity & $20(24.1)$ & $86(23.8)$ & & $106(23.8)$ \\
\hline Hypopharynx & $14(17.0)$ & $50(13.8)$ & & $64(14.3)$ \\
\hline Larynx & $15(18.1)$ & $42(11.6)$ & & $57(12.8)$ \\
\hline Others $^{a}$ & $12(14.5)$ & $15(4.1)$ & & $27(6.1)$ \\
\hline Histologic grade & & & 0.109 & \\
\hline Well differentiated & $11(13.3)$ & $57(15.8)$ & & $68(15.3)$ \\
\hline Moderate differentiated & $27(32.6)$ & $132(36.6)$ & & $159(35.7)$ \\
\hline Poorly differentiated & $7(8.4)$ & $59(16.3)$ & & $66(14.8)$ \\
\hline Not assessed & $38(45.7)$ & $114(31.5)$ & & $152(34.1)$ \\
\hline T classification & & & 0.018 & \\
\hline $\mathrm{T} 1$ & $13(15.7)$ & $53(14.6)$ & & $66(14.8)$ \\
\hline $\mathrm{T} 2$ & $26(31.3)$ & $146(40.3)$ & & $172(38.7)$ \\
\hline T3 & $18(21.7)$ & $76(21.0)$ & & $94(21.1)$ \\
\hline $\mathrm{T} 4 \mathrm{a} / \mathrm{T} 4 \mathrm{~b}$ & $17 / 9(31.3)$ & $76 / 9(23.5)$ & & $93 / 18(24.9)$ \\
\hline Unknown & 0 & $2(0.6)$ & & $2(0.5)$ \\
\hline N classification & & & 0.086 & \\
\hline NO & $12(14.5)$ & $40(11.1)$ & & $52(11.7)$ \\
\hline N1 & $21(25.3)$ & $119(32.9)$ & & $140(31.5)$ \\
\hline N2 & $49(59.0)$ & $196(54.1)$ & & $245(55.1)$ \\
\hline N3 & 0 & $7(1.9)$ & & $7(1.6)$ \\
\hline Unknown & $1(1.2)$ & 0 & & $1(0.2)$ \\
\hline P16/HPV status & & & 0.032 & \\
\hline Negative & 15 (18.1) & $84(23.3)$ & & $99(22.3)$ \\
\hline Positive & $10(12.0)$ & $80(22.1)$ & & $90(20.2)$ \\
\hline Unknown & $58(69.9)$ & $198(54.7)$ & & $256(57.5)$ \\
\hline
\end{tabular}

Values are presented as median (range) or number (\%).

ECOG, Eastern Cooperative Oncology Group; PS, performance status; HPV, human papillomavirus.

${ }^{a}$ Sinus, unknown primary site, double primary sites. 


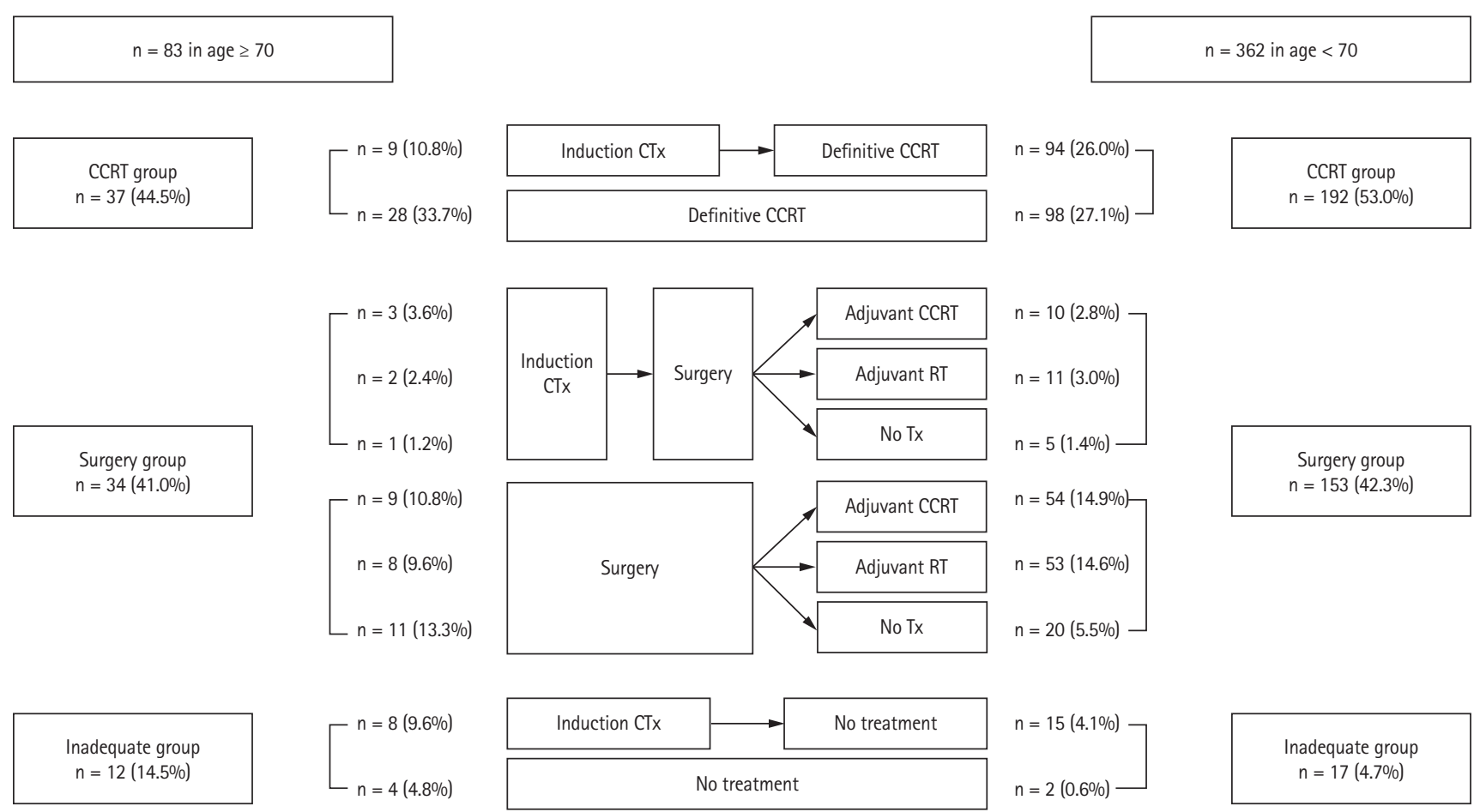

Figure 1. Flowchart for the treatment of locally advanced head and neck squamous cell carcinoma by age of 70 ( $n=445)$. CCRT, concurrent chemoradiotherapy; CTx, chemotherapy; RT, radiotherapy; Tx, treatment.

ceiving IC did not receive subsequent therapy (Fig. 1 in inadequate group). Approximately $72 \%$ of older patients and $90 \%$ of younger patients underwent combined treatment modalities.

\section{Treatment characteristics}

Treatment characteristics are summarized in Table 2. Among 158 patients receiving IC, 23 (14.6\%) were older. For IC regimen, the choice of chemotherapy differed according to 70 years of age $(p<0.001)$. In older patients, fluorouracil and cisplatin (FP) was the most preferred (47.9\%) regimen. However, docetaxel and cisplatin (DP) was the most chosen IC regimen in patients aged $<70$ years. Overall treatment response was similar between the age groups $(p=0.448)$; complete response, partial response, and stable disease were $17.4 \%, 47.8 \%$, and $30.4 \%$, respectively, in older patients and $15.6 \%, 56.3 \%$, and $17.8 \%$, respectively, in younger patients.

Among the 229 patients receiving definitive CCRT, the chemotherapy regimen was not different between the age groups ( $p=0.497$ ). The preferred regimen was weekly cis- platin in $62.2 \%$ of older patients and $57.3 \%$ of younger patients. Among the 11 patients receiving other regimens, six received cetuximab (two were older and four were younger patients). The overall response of definitive CCRT was similar between older and younger patients $(p=0.534)$.

\section{Study outcomes}

For 445 LA-HNSCC patients, 113 deaths were observed with a median follow-up period of 39.3 months (95\% confidence interval [Cl], 35.4 to 43.1). For 83 older patients, 26 (31.3\%) patients died with a median follow-up time of 30.8 months ( $95 \% \mathrm{Cl}, 24.6$ to 44.3$)$. The older patients had a significantly shorter median OS than younger patients (65.5 months vs. not reached: hazard ratio [HR], 1.60; $95 \% \mathrm{Cl}, 1.03$ to 2.49; $p=0.035$ ) (Fig. 2A). The 12- and 24-month survival rates were $84.5 \%(95 \% \mathrm{Cl}, 0.74$ to 0.91$)$ and $75.1 \%(95 \% \mathrm{Cl}$, 0.63 to 0.84$)$ in older patients, and $89.6 \%(95 \% \mathrm{Cl}, 0.86$ to 0.92$)$ and $80.8 \%(95 \% \mathrm{Cl}, 0.76$ to 0.85$)$ in younger patients, respectively. Regarding OS in the context of primary tumor location, median OS was 13.4 months $(95 \% \mathrm{Cl}, 5.4$ to not reached) in oral cavity, 49.6 months $(95 \% \mathrm{Cl}, 24.8$ to 
Table 2. Characteristics of therapeutics with locally advanced head and neck squamous cell carcinoma by age group

\begin{tabular}{|c|c|c|c|c|}
\hline \multirow{2}{*}{ Treatment } & \multicolumn{2}{|c|}{ Age group, yr } & \multirow{2}{*}{$p$ value } & \multirow{2}{*}{ Total } \\
\hline & Age $\geq 70$ & Age $<70$ & & \\
\hline Induction chemotherapy & $23(14.6)$ & $135(85.4)$ & 0.100 & 158 \\
\hline Regimen & & & $<0.001$ & \\
\hline Docetaxel + Cisplatin & $10(43.4)$ & $67(49.6)$ & & $77(48.7)$ \\
\hline Docetaxel + Cisplatin + Fluorouracil & $1(4.4)$ & $41(30.4)$ & & $42(26.6)$ \\
\hline Fluorouracil + Cisplatin & $11(47.8)$ & $17(12.6)$ & & $28(17.7)$ \\
\hline Others $^{\mathrm{a}}$ & $1(4.4)$ & $10(7.4)$ & & $11(7.0)$ \\
\hline No. of cycles & $3(1-4)$ & $3(1-4)$ & 0.661 & $3(1-4)$ \\
\hline Best overall response & & & 0.448 & \\
\hline Complete response & $4(17.4)$ & $21(15.6)$ & & $25(15.8)$ \\
\hline Partial response & $11(47.8)$ & $76(56.3)$ & & $87(55.1)$ \\
\hline Stable disease & $7(30.4)$ & $24(17.8)$ & & $31(19.6)$ \\
\hline Progressive disease & $1(4.4)$ & $14(10.4)$ & & $15(9.5)$ \\
\hline Definitive concurrent chemoradiotherapy & $37(16.2)$ & $192(83.8)$ & 0.119 & 229 \\
\hline Regimen & & & 0.497 & \\
\hline Weekly cisplatin & $23(62.2)$ & $110(57.3)$ & & $133(58.1)$ \\
\hline 3-weekly cisplatin & $7(18.9)$ & $56(29.2)$ & & $63(27.5)$ \\
\hline Fluorouracil + Cisplatin & $4(10.9)$ & $18(9.4)$ & & $22(9.6)$ \\
\hline Others $^{b}$ & $3(8.1)$ & $8(4.2)$ & & $11(4.8)$ \\
\hline Total radiation dose, Gy & $67.5 \pm 12.8$ & $67.5 \pm 11.1$ & 0.440 & $67.5 \pm 11.4$ \\
\hline Best overall response & & & 0.534 & \\
\hline Complete response & $22(59.5)$ & $126(65.6)$ & & $148(6.5)$ \\
\hline Partial response & $10(27.0)$ & $32(16.7)$ & & $42(18.5)$ \\
\hline Stable disease & $3(8.1)$ & $18(9.4)$ & & $21(9.3)$ \\
\hline Progressive disease & $2(5.4)$ & $14(7.3)$ & & $16(7.1)$ \\
\hline Not evaluable & 0 & $2(1.0)$ & & \\
\hline
\end{tabular}

Values are presented as number (\%), median (range), or mean \pm standard deviation.

${ }^{a}$ Age $\geq 70$ : cetuximab + docetaxel + cisplatin in 1 patient; Age $<70:$ TS- 1 + docetaxel + cisplatin in 8 patients, docetaxel in 1 patient, cisplatin in 1 patient.

${ }^{\mathrm{b}}$ Age $\geq 70$ : cetuximab in 2 patients, weekly carboplatin in 1 patient; Age < 70: cetuximab in 4 patients, weekly carboplatin in 4 patients.

not reached), 65.5 months ( $95 \% \mathrm{Cl}, 65.5$ to not reached) in hypopharynx, and not reached in oropharynx: older patients with oral cavity tumors had the worst probability of survival ( $p=0.03)$ (Fig. 2B).

IC administration did not affect median OS (not reached in IC vs. 65.5 months without IC) in older patients (HR, 0.75; $95 \% \mathrm{Cl}, 0.30$ to $1.89 ; p=0.547$ ) (Fig. 2C). Regarding treatment strategies in older patients, the 12- and 24-month survival rates were $88.3 \%(95 \% \mathrm{Cl}, 0.72$ to 0.95$)$ and $80.8 \%$
(95\% Cl, 0.62 to 0.92$)$ in CCRT group, $87.5 \%(95 \% \mathrm{Cl}$, 0.70 to 0.95$)$ and $77.4 \%(95 \% \mathrm{Cl}, 0.58$ to 0.89$)$ in surgery group, and $65.6 \%(95 \% \mathrm{Cl}, 0.32$ to 0.86$)$ and $52.5 \%(95 \%$ $\mathrm{Cl}, 0.19$ to 0.78$)$ in inadequate group, respectively. Survival probabilities were not significantly different between those receiving CCRT and those undergoing surgery (HR, 1.61; $95 \% \mathrm{Cl}, 0.68$ to $3.83 ; p=0.283$ ) (Fig. 2D). Patients who did not undergo adequate treatments had the poorest OS. 

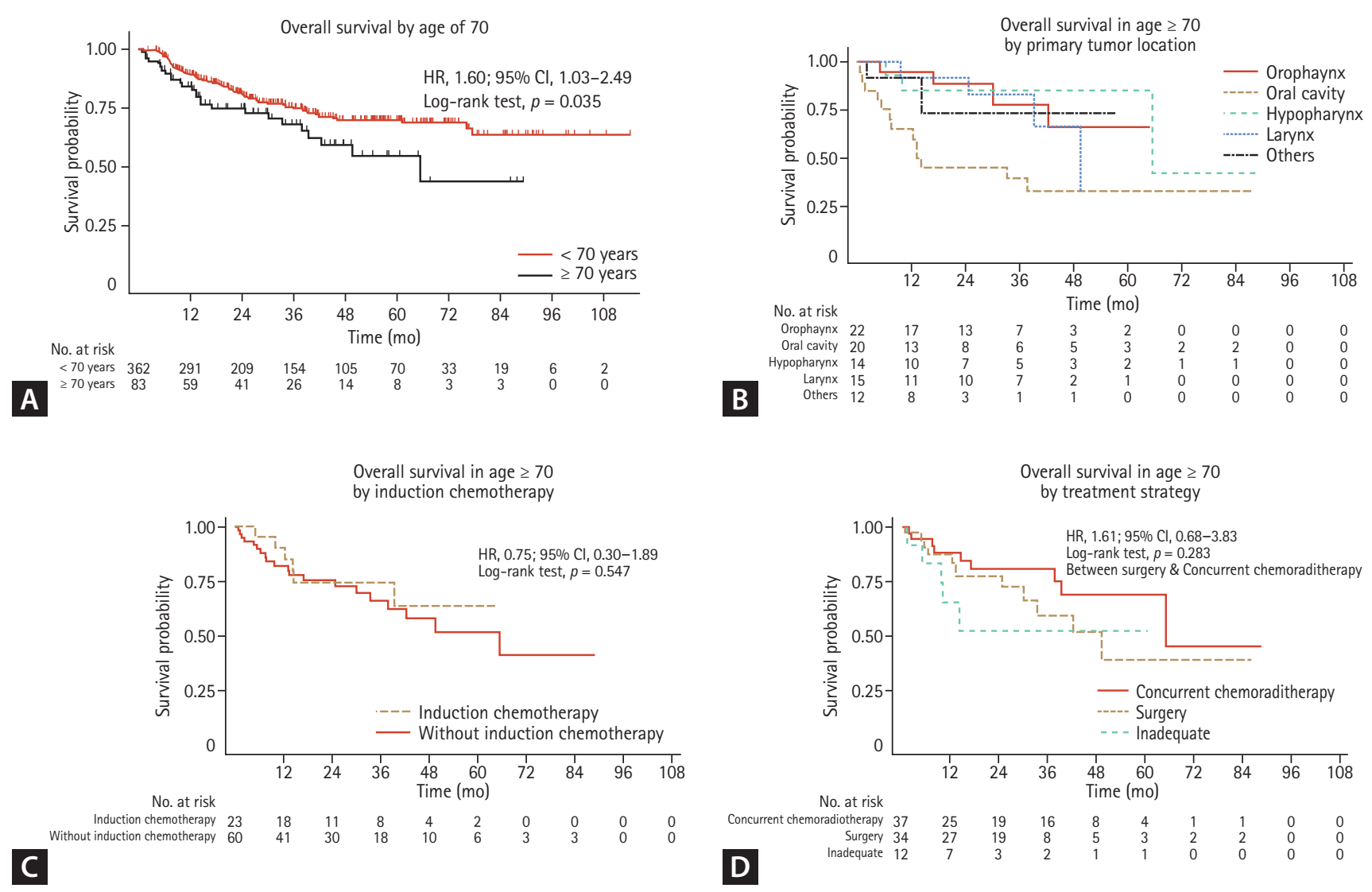

Figure 2. (A) Overall survival by age of $70(n=445)$. (B) Overall survival by primary tumor site in age $\geq 70(n=83)$. (C) Overall survival by induction chemotherapy in age $\geq 70(n=83)$. (D) Overall survival by treatment strategy in age $\geq 70(n=83)$. HR, hazard ratio; $C l$, confidence interval.

Table 3. Multivariate analyses for risk factors of overall survival by age of 70

\begin{tabular}{lcc}
\hline Outcome & Estimate HR $(95 \% \mathrm{Cl})$ & $p$ value \\
\hline Overall survival, age $\geq 70$ years $(n=83)$ & & 0.002 \\
$\quad$ Primary location (oral cavity vs. oropharynx) & $3.33(1.54-7.22)$ & 0.006 \\
\hline Overall survival, age $<70$ years $(n=362)$ & $1.36(1.09-1.70)$ & 0.001 \\
\hline T classification (from one unit to next) & $1.66(1.22-2.26)$ & 0.008 \\
N classification (from one unit to next) & $0.32(0.14-0.74)$ & 0.014 \\
\hline HPV status (positive vs. negative) & $2.28(1.18-4.37)$ & \\
\hline Treatment strategy (inadequate vs. CCRT) & & \\
\hline
\end{tabular}

$\mathrm{HR}$, hazard ratio; $\mathrm{Cl}$, confidence interval; HPV, human papillomavirus; CCRT, concurrent chemoradiotherapy.

\section{Multivariate analyses of os}

In each age group, uni- and multivariate analyses identified different survival-related risk factors (Table 3, Supplementary Tables 1 and 2). In older patients, oral cavity primary tumor location (compared with the oropharynx) was a strong and independent predictor for poor OS (HR, 3.33; $95 \% \mathrm{Cl}$, 1.54 to $7.22 ; p=0.002$ ) (Table 3, Supplementary Table 1). In contrast, in younger patients, tumor factors, including advanced T or N stage, and HPV status were independent prognostic factors. Additionally, the failure to receive an ad- 
equate treatment was a strong predictor for poor OS (HR, 2.28; $95 \% \mathrm{Cl}, 1.18$ to $4.37 ; p=0.014$ ) (Table 3, Supplementary Table 2).

\section{DISCUSSION}

In this retrospective nationwide investigation of a Korean cohort of patients with stage III-IV LA-HNSCC, older patients accounted for $18.7 \%$ (83/445) of all patients. Compared with younger patients, older patients have poor clinical characteristics and poor survival probabilities. The proportion of advanced T stage or poor PS was higher among older patients than among younger patients, while the proportion of older patients with oropharyngeal cancer or HPV positive cancer was lower than that of younger patients. In particular, older patients with oral cavity cancer had the worst OS. In previous epidemiological studies on geriatric patients, a greater proportion of oral cavity or larynx tumors were reported, but the proportion of oropharyngeal cancer was low $[4,5,11]$. Moreover, patients with oral cavity cancer, especially the older population, had a significantly poor survival [12]. In addition, although HPV is known to be a good prognostic factor in younger patients with oropharyngeal cancer, it is infrequently reported in older patients, which is also consistent with the results of this study [13-15]. Altogether, the evidence indicates that older patients have poor prognostic tumor factors (Fig. 2A).

Treatment patterns were similar between the two age groups ( $\geq 70$ years vs. $<70$ years) (Fig. 1). There was no survival difference according to treatment modalities such as CCRT or surgery (Fig. 2D) or administration of IC (Fig. 2C). The proportion of older patients who completed their planned treatments was lower than that of younger patients (Fig. 1). The survival of older patients who did not finish their planned treatments tended to be poor (Table 3).

Still, the role of age as a prognostic factor is not clearly defined in LA-HNSCC. Previous retrospective or epidemiologic-based analysis of various tumor status or treatment conditions revealed that chronologic age was not a significant prognostic factor [16-18]. Furthermore, there is limited data on the survival of older LA-HNSCC patients undergoing multi-modal treatments. In a large-sized study using a cancer registry, older patients aged $\geq 70$ years had a two-fold poorer survival than younger aged patients. In a subgroup analysis of patients with stage III or IV larynge- al cancer, patients who received single modality treatment had extremely poor survival than all other patients [19]. In fact, in the real-world practice, LA-HNSCC patients aged $\geq$ 70 years tend to receive less-aggressive strategies [20]. In our analysis, older patients received weaker IC and CCRT intensity regimens. Eight of 12 patients who received IC did not receive definitive treatment. The failure to complete planned treatments among older patients was associated with a poor survival tendency, although owing to the small number of patients, this difference was not statistically significant. Consequently, in our study, treatment-related factors and poor tumor-related factors among older patients might contribute to poor survival outcomes compared with those among younger patients.

In the clinical practice, treatment strategies for LA-HNSCC are decided through multidisciplinary consultation and considering multifaceted clinical factors. In our analysis, surgery and definitive CCRT as primary treatment was performed in $41.0 \%$ and $44.6 \%$ of older patients, respectively. As expected, there was no significant difference in survival with each treatment strategy. Approximately $27.7 \%$ (23/83) of older patients received IC as initial treatment. Among them, upfront administration of IC did not show statistical difference in survival. There were no significant differences in survival among treatment modalities; however, there were differences especially in application of chemotherapeutic agents in ICT and CCRT for older patients comparing with younger patients.

In the older group, only one patient received docetaxel, cisplatin, and 5-fluorouracil (TPF) as IC treatment, which is regarded as a standard regimen of IC, whereas $30.4 \%$ of younger patients who received IC were treated with TPF. Most (91.3\%) older patients received doublet regimens such as DP or FP for IC. Though the intensity of doublet regimen might be weaker than triple regimen of TPF, the facts that substantial portion of older patients receiving IC did not continue further definitive treatment suggest the physician need to focus on safety rather than the effectiveness of specific treatments.

The reason of the majority of patients who received IC were treated with doublet regimen is suspected from oncologist's concern for frailty of older patients. To obtain the best efficacy through IC, three cycles of treatment with TPF is the strong recommended regimen because IC using TPF regimen provided survival benefit compared to FP in phase III clinical trials and meta-analysis [21-24]. However, TPF is- 
sued because of its toxicities. TPF showed more incidence of grade 3-4 neutropenia, febrile neutropenia and neutropenic infection compared to FP $[22,23]$. In addition, IC with TPF is discussed because it could compromise the following treatment because of toxicity. In a CONDOR study, only $22 \%$ of the patients received CCRT with cumulative dose of cisplatin $>200 \mathrm{mg} / \mathrm{m}^{2}$ after three cycles of IC with TPF [25]. Also, in a phase III trial that compared TPF-IC or FPIC followed by CCRT versus definitive CCRT alone, a lower proportion of patients in the TPF-IC arm could receive CCRT than that of patients in the FP-IC arm [26]. Such results indicate that IC using triplet regimen can decrease the chances of receiving CCRT and also decrease the dose of therapeutic cisplatin combined with CCRT. Serious adverse events occurring during IC can affect further treatment or decrease treatment intensity, which consequently can affect patients' chances of being cured. However, it is important to note that in our analysis, despite the low intensity of IC, survival did not differ between the IC and non-IC groups. Therefore, IC with doublet regimen rather than triple regimen is recommendable for older patients. Given the lack of studies regarding the best $\mathrm{IC}$ regimen for older patients, a well-organized study aiming to identify the most suitable IC regimen for older patients is needed to avoid unnecessary chemotherapy-related toxicities and achieve better treatment outcomes.

In a group of definitive CCRT, $24.3 \%$ (9/37) of older patients received CCRT after IC and 75.7\% (28/37) received definitive CCRT as the primary treatment. There was no significant difference in selecting a concomitant chemotherapy regimen between older and younger patients in Korea. However, meaningful differences in the efficacy between a weekly or 3-weekly cisplatin during CCRT are not yet clearly defined. In a previous retrospective study conducted in Korea, weekly cisplatin showed comparable therapeutic outcomes compared with 3-weekly cisplatin [27]. Because weekly cisplatin regimen is more compliant and has less toxicities related to myelosuppression, nephrotoxicity, and emesis, weekly cisplatin might be more considerable in older patients [28]. In addition, survival of patients who received CCRT was not different from that of patients who underwent surgery.

This study had some limitations. First, this study was retrospectively designed; therefore, exact information regarding comorbidities, treatment toxicities, and quality of life could not be obtained. Thus, we could not evaluate the correla- tion between these factors and treatment results in detail. However, because LA-HNSCC patient data for this study were retrieved from 13 nationwide referral hospitals, the data reflect of real treatment patterns for older patients in Korea. Second, patients with various primary sites of head and neck cancer were included, but interpretation of specific cancer status, especially regarding HPV positivity, needs to be performed with caution because we could only obtain information on HPV status in $30.1 \%$ of patients. In conclusion, we defined poor tumor characteristics and real-world treatment patterns of older LA-HNSCC patients in Korea. Older LA-HNSCC patients had aggressive tumor characteristics and received less intensive treatment, which resulted in poor survival, especially in cases of oral cavity cancer. There were no survival differences among treatment modalities. Future research focusing on older patients is necessary to determine an optimal treatment strategy that can improve survival.

\section{KEY MESSAGE}

1. Older locally advanced (LA)-head and neck squamous cell carcinoma (HNSCC) patients had aggressive tumor characteristics.

2. Older LA-HNSCC patients received less intensive treatments during concurrent chemoradiotherapy or induction chemotherapy.

3. Older LA-HNSCC patients had a higher risk of death comparing to younger patients especially in patients with oral cavity cancer.

\section{Conflict of interest}

No potential conflict of interest relevant to this article was reported.

\section{Acknowledgments}

This study was supported by a grant from the National R\&D Program for Cancer Control, Ministry of Health and Welfare, Republic of Korea (HA16C0015) (1720150). The research was supported (in part) by the Korean Cancer Study Group (KCSG) and KCSG data center. We thank Jiyun Mun from the KCSG data center. 


\section{REFERENCES}

1. Bray F, Ferlay J, Soerjomataram I, Siegel RL, Torre LA, Jemal A. Global cancer statistics 2018: GLOBOCAN estimates of incidence and mortality worldwide for 36 cancers in 185 countries. CA Cancer J Clin 2018;68:394-424.

2. Jung KW, Won YJ, Kong HJ, Lee ES. Cancer statistics in Korea: incidence, mortality, survival, and prevalence in 2016. Cancer Res Treat 2019;51:417-430.

3. National Cancer Statistics Korea. Annual report of cancer statistics in Korea in 2018 [Internet]. Goyang (KR): National Cancer Statistics Korea, 2021 [cited 2021 Nov 22]. Available from: https://ncc.re.kr/cancerStatsView.ncc?bbsnum =558\&searchKey=total\&searchValue=\&pageNum $=1$.

4. Kruse AL, Bredell M, Luebbers HT, Gratz KW. Head and neck cancer in the elderly: a retrospective study over 10 years (1999-2008). Head Neck Oncol 2010;2:25.

5. Huang SH, O'Sullivan B, Waldron J, et al. Patterns of care in elderly head-and-neck cancer radiation oncology patients: a single-center cohort study. Int J Radiat Oncol Biol Phys 2011;79:46-51.

6. Noor A, Gibb C, Boase S, Hodge JC, Krishnan S, Foreman A. Frailty in geriatric head and neck cancer: a contemporary review. Laryngoscope 2018;128:E416-E424.

7. Paleri $\mathrm{V}$, Wight RG, Silver $C E$, et al. Comorbidity in head and neck cancer: a critical appraisal and recommendations for practice. Oral Oncol 2010;46:712-719.

8. Derks W, de Leeuw RJ, Hordijk GJ. Elderly patients with head and neck cancer: the influence of comorbidity on choice of therapy, complication rate, and survival. Curr Opin Otolaryngol Head Neck Surg 2005;13:92-96.

9. Pallis AG, Fortpied C, Wedding $U$, et al. EORTC elderly task force position paper: approach to the older cancer patient. Eur J Cancer 2010;46:1502-1513.

10. Balducci L. Geriatric oncology: challenges for the new century. Eur J Cancer 2000;36:1741-1754.

11. Italiano A, Ortholan C, Dassonville $O$, et al. Head and neck squamous cell carcinoma in patients aged $>$ or $=80$ years: patterns of care and survival. Cancer 2008;113:3160-3168.

12. Barros-Silva PG, Fontes-Borges MM, Costa-Dias C, et al. Clinical-pathological and sociodemographic factors associated with the distant metastasis and overall survival of oral cavity and oropharynx squamous cell carcinoma. Med Oral Patol Oral Cir Bucal 2020;25:e375-e382.

13. Wagner S, Sharma SJ, Wuerdemann N, et al. Human papillomavirus-related head and neck cancer. Oncol Res Treat
2017;40:334-340.

14. Rettig EM, D'Souza G. Epidemiology of head and neck cancer. Surg Oncol Clin N Am 2015;24:379-396.

15. Chaturvedi AK, Anderson WF, Lortet-Tieulent J, et al. Worldwide trends in incidence rates for oral cavity and oropharyngeal cancers. J Clin Oncol 2013;31:4550-4559.

16. Bhattacharyya N. A matched survival analysis for squamous cell carcinoma of the head and neck in the elderly. Laryngoscope 2003;113:368-372.

17. Argiris A, Li Y, Murphy BA, Langer CJ, Forastiere AA. Outcome of elderly patients with recurrent or metastatic head and neck cancer treated with cisplatin-based chemotherapy. J Clin Oncol 2004;22:262-268.

18. Lusinchi A, Bourhis J, Wibault P, Le Ridant AM, Eschwege F. Radiation therapy for head and neck cancers in the elderly. Int J Radiat Oncol Biol Phys 1990;18:819-823.

19. Moye VA, Chandramouleeswaran S, Zhao N, et al. Elderly patients with squamous cell carcinoma of the head and neck and the benefit of multimodality therapy. Oncologist 2015;20:159-165.

20. Juarez JE, Choi J, St John M, Abemayor E, TenNapel M, Chen AM. Patterns of care for elderly patients with locally advanced head and neck cancer. Int J Radiat Oncol Biol Phys 2017;98:767-774.

21. Vermorken JB, Remenar E, van Herpen C, et al. Cisplatin, fluorouracil, and docetaxel in unresectable head and neck cancer. N Engl J Med 2007;357:1695-1704.

22. Lorch JH, Goloubeva O, Haddad RI, et al. Induction chemotherapy with cisplatin and fluorouracil alone or in combination with docetaxel in locally advanced squamous-cell cancer of the head and neck: long-term results of the TAX 324 randomised phase 3 trial. Lancet Oncol 2011;12:153-159.

23. Posner MR, Hershock DM, Blajman CR, et al. Cisplatin and fluorouracil alone or with docetaxel in head and neck cancer. N Engl J Med 2007;357:1705-1715.

24. Blanchard P, Bourhis J, Lacas B, et al. Taxane-cisplatin-fluorouracil as induction chemotherapy in locally advanced head and neck cancers: an individual patient data meta-analysis of the meta-analysis of chemotherapy in head and neck cancer group. J Clin Oncol 2013;31:2854-2860.

25. Driessen CM, de Boer JP, Gelderblom H, et al. Induction chemotherapy with docetaxel/cisplatin/5-fluorouracil followed by randomization to two cisplatin-based concomitant chemoradiotherapy schedules in patients with locally advanced head and neck cancer (CONDOR study) (Dutch Head and Neck Society 08-01): a randomized phase II study. Eur J Cancer 
2016:52:77-84.

26. Hitt R, Grau JJ, Lopez-Pousa A, et al. A randomized phase III trial comparing induction chemotherapy followed by chemoradiotherapy versus chemoradiotherapy alone as treatment of unresectable head and neck cancer. Ann Oncol 2014;25:216225.

27. Lee SY, Choi YS, Song IC, et al. Comparison of standard-dose 3-weekly cisplatin and low-dose weekly cisplatin for concur- rent chemoradiation of patients with locally advanced head and neck squamous cell cancer: a multicenter retrospective analysis. Medicine (Baltimore) 2018;97:e10778.

28. Szturz P, Wouters K, Kiyota N, et al. Weekly low-dose versus three-weekly high-dose cisplatin for concurrent chemoradiation in locoregionally advanced non-nasopharyngeal head and neck cancer: a systematic review and meta-analysis of aggregate data. Oncologist 2017;22:1056-1066. 
Kang EJ, et al. Treatment in older head and neck cancer

Supplementary Table 1. Univariate and multivariate analyses for overall survival in 83 patients in age $\geq 70$

\begin{tabular}{|c|c|c|c|c|}
\hline \multirow{2}{*}{ Characteristic } & \multicolumn{2}{|c|}{ Univariate } & \multicolumn{2}{|c|}{ Multivariate } \\
\hline & $\mathrm{HR}(95 \% \mathrm{Cl})$ & $p$ value & $\mathrm{HR}(95 \% \mathrm{Cl})$ & $p$ value \\
\hline \multicolumn{5}{|l|}{ Gender } \\
\hline Male vs. female & Incalculable & 1.00 & & \\
\hline \multicolumn{5}{|l|}{ ECOG PS } \\
\hline $2-3$ vs. $0-1$ & $2.39(0.71-8.02)$ & 0.157 & & \\
\hline \multicolumn{5}{|l|}{ Smoking history } \\
\hline Current or former vs. never & $1.31(0.48-3.62)$ & 0.597 & & \\
\hline \multicolumn{5}{|l|}{ Alcohol history } \\
\hline Drink vs. Do not drink & $1.21(0.48-3.07)$ & 0.689 & & \\
\hline \multicolumn{5}{|l|}{ HPV status } \\
\hline Positive vs. Negative & $0.18(0.02-1.45)$ & 0.106 & & \\
\hline Unknown vs. Negative & $0.53(0.22-1.28)$ & 0.156 & & \\
\hline Primary tumor location & & & & 0.002 \\
\hline Oropharynx & 1 (reference) & & 1 (reference) & \\
\hline Oral cavity & $3.83(1.24-11.85)$ & 0.020 & $3.33(1.54-7.22)$ & \\
\hline Hypopharynx & $1.06(0.23-4.75)$ & 0.943 & & \\
\hline Larynx & $1.35(0.34-5.40)$ & 0.675 & & \\
\hline Others & $1.26(0.23-2.97)$ & 0.787 & & \\
\hline T classification & $1.06(0.74-1.53)$ & 0.736 & & \\
\hline N classification & $1.04(0.60-1.80)$ & 0.897 & & \\
\hline \multicolumn{5}{|l|}{ Induction chemotherapy } \\
\hline Yes vs. No & $0.75(0.30-1.89)$ & 0.547 & & \\
\hline \multicolumn{5}{|l|}{ Treatment strategy } \\
\hline CCRT & 1 (reference) & & & \\
\hline Surgery & $1.61(0.68-3.83)$ & 0.283 & & \\
\hline Inadequate & $2.40(0.79-7.25)$ & 0.121 & & \\
\hline
\end{tabular}

HR, hazard ratio; $\mathrm{Cl}$, confidence interval; ECOG, Eastern Cooperative Oncology Group; PS, performance status; HPV, human papilloma virus; CCRT, concurrent chemoradiotherapy. 
Supplementary Table 2. Univariate and multivariate analyses for overall survival in 362 patients in age $<70$

\begin{tabular}{|c|c|c|c|c|}
\hline \multirow{2}{*}{ Characteristic } & \multicolumn{2}{|c|}{ Univariate } & \multicolumn{2}{|c|}{ Multivariate } \\
\hline & $\mathrm{HR}(95 \% \mathrm{Cl})$ & $p$ value & $\mathrm{HR}(95 \% \mathrm{CI})$ & $p$ value \\
\hline \multicolumn{5}{|l|}{ Gender } \\
\hline Male vs. female & $0.80(0.46-1.40)$ & 0.431 & & \\
\hline \multicolumn{5}{|l|}{ ECOG PS } \\
\hline $2-3$ vs. $0-1$ & $1.92(0.77-4.79)$ & 0.160 & & \\
\hline \multicolumn{5}{|l|}{ Smoking history } \\
\hline Current or former vs. never & $1.04(0.63-1.73)$ & 0.876 & & \\
\hline \multicolumn{5}{|l|}{ Alcohol history } \\
\hline Drink vs. Do not drink & $1.25(0.72-2.18)$ & 0.419 & & \\
\hline \multicolumn{5}{|l|}{ HPV status } \\
\hline Positive vs. Negative & $0.27(0.11-0.67)$ & 0.005 & $0.32(0.14-0.74)$ & 0.008 \\
\hline Unknown vs. Negative & $1.06(0.65-1.72)$ & 0.817 & & \\
\hline \multicolumn{5}{|l|}{ Primary tumor location } \\
\hline Oropharynx & 1 (reference) & & & \\
\hline Oral cavity & $1.41(0.84-2.37)$ & 0.195 & & \\
\hline Hypopharynx & $1.92(1.06-3.50)$ & 0.032 & & \\
\hline Larynx & $0.94(0.45-1.96)$ & 0.860 & & \\
\hline Others & $1.13(0.40-3.19)$ & 0.821 & & \\
\hline T classification & $1.50(1.21-1.86)$ & $<0.001$ & $1.36(1.09-1.70)$ & 0.006 \\
\hline N classification & $1.70(1.22-2.36)$ & 0.002 & $1.66(1.22-2.26)$ & 0.001 \\
\hline \multicolumn{5}{|l|}{ Induction chemotherapy } \\
\hline Yes vs. No & $1.82(1.19-2.77)$ & 0.005 & & \\
\hline \multicolumn{5}{|l|}{ Treatment strategy } \\
\hline CCRT & 1 (reference) & & 1 (reference) & \\
\hline Surgery & $0.79(0.50-1.25)$ & 0.314 & & \\
\hline Inadequate & $2.96(1.52-5.77)$ & 0.001 & $2.28(1.18-4.37)$ & 0.014 \\
\hline
\end{tabular}

HR, hazard ratio; Cl, confidence interval; ECOG, Eastern Cooperative Oncology Group; PS, performance status; HPV, human papilloma virus; CCRT, concurrent chemoradiotherapy. 